\title{
Ankle-Brachial Index in Systemic Lupus Erythematosus: A Senegalese Case-Control Study
}

\author{
Baïdy Sy Kane', Maïmouna Sow ${ }^{1}$, Fatou Aw ${ }^{2}$, Abdourahmane Samba ${ }^{3}$, Ahmed Tall Lemrabott ${ }^{4}$, \\ Awa Cheikh Ndao', Souhaibou Ndongo', Mouhamadou Bamba Ndiaye ${ }^{2}$, Maboury Diao², \\ Abdoulaye Pouye ${ }^{1}$
}

\begin{abstract}
${ }^{1}$ Department of Internal Medicine, Le Dantec Teaching Hospital, Cheikh Anta DIOP University, Dakar, Senegal
${ }^{2}$ Department of Cardiology, Le Dantec Teaching Hospital, Cheikh Anta DIOP University, Dakar, Senegal

${ }^{3}$ Biochemistry Laboratory, Le Dantec Teaching Hospital, Cheikh Anta DIOP University, Dakar, Senegal

${ }^{4}$ Department of Nephrology, Le Dantec Teaching Hospital, Cheikh Anta DIOP University, Dakar, Senegal

Email: baidykane@gmail.com
\end{abstract}

How to cite this paper: Kane, B.S., Sow, M., Aw, F., Samba, A., Lemrabott, A.T., Ndao, A.C., Ndongo, S., Ndiaye, M.B., Diao, M. and Pouye, A. (2019) Ankle-Brachial Index in Systemic Lupus Erythematosus: A Senegalese Case-Control Study. World Journal of Cardiovascular Diseases, 9, 958-968.

https://doi.org/10.4236/wjcd.2019.912084

Received: December 4, 2019

Accepted: December 24, 2019

Published: December 27, 2019

Copyright $\odot 2019$ by author(s) and Scientific Research Publishing Inc. This work is licensed under the Creative Commons Attribution International License (CC BY 4.0).

http://creativecommons.org/licenses/by/4.0/

\begin{abstract}
Systemic lupus erythematosus (SLE) is associated with accelerated atherosclerosis and increasing cardiovascular risk which is recognized as a major cause of morbidity and death. Whether subclinical atherosclerosis has been evaluated by several methods, there are very limited data about Ankle-Brachial Index (ABI) in patients with systemic lupus erythematosus. The aim of the present study was to compare this index, between SLE patients and controls. We conducted a case-control study in the Department of Internal Medicine of our institution during the period from August 1, 2017 to December 31, 2018. We included 100 subjects, including 50 SLE patients and 50 control cases. This included 44 women and 6 men in patients with an identical distribution in controls. The mean age was $33.5 \pm 11.3$ years for cases and $33.3 \pm 11.3$ years (p-value: 0.93 ) for controls subjects. There was higher frequency of cardiovascular risk factors such as dyslipidemia ( $\mathrm{p}$-value at 0.009), low level of serum HDL-cholesterol ( $\mathrm{p}$-value $<0.001$ ), hypertriglyceridemia (p-value at 0.000 ) and hyperuricemia (p-value at 0.000 ) in patients with SLE. Overweight/obesity was higher in controls subjects ( $\mathrm{p}$-value at 0.028 ). There was no statistically significant difference in the frequency of diabetes-mellitus, smoking and high blood pressure. The abnormally ABI was recorded in 19 patients with SLE (38\%) and 8 controls (16\%) with a p-value: 0.01 and odds ratio: 3.22. Eight patients (16\%) and four controls (8\%) had low ABI without significant difference (p-value: 0.11 but odds-ratio at 2.98). Eleven patients with SLE (22\%) and five controls (10\%) had high ABI (p-value: 0.05 and odds-ratio: 3.24). In patients with SLE only disease activity (cSLEDAI) at the inclusion of the study was correlated to abnormal ABI. Conclusion: This
\end{abstract}


study showed an increasing prevalence of abnormal ABI in black African patients with SLE compared to controls with a correlation between disease activity and abnormal ABI.

\section{Keywords}

Systemic Lupus Erythematosus, Subclinical Atherosclerosis, Peripheral Arterial Disease, Ankle-Brachial Index, Africa South of the Sahara

\section{Introduction}

Systemic Lupus Erythematosus (SLE) is a complex systemic auto-immune disease characterized by a wide spectrum of clinical, laboratory and immunological abnormalities and a variable course and outcome [1].

SLE is associated with accelerated atherosclerosis and increasing cardiovascular risk which is recognized as a major cause of morbidity and death [2]-[7].

Traditional cardiovascular factors (including Diabetes mellitus, hypertension, tobacco, atherogenic dyslipidemia), SLE therapies such as corticosteroid and disease-related factors (subclinical inflammation, oxidative stress, antiphospholipid antibodies) appear to contribute to the increasing of incidence of cardiovascular events in SLE [5] [8] [9] [10].

A variety of modalities have been used recently in several studies to investigate endothelial dysfunction, subclinical cardiovascular disease (CVD) or to predict cardiovascular risk (measurement of intima-media thickness and detection of plaques by ultrasound) [11] [12] [13]. Another noninvasive marker of subclinical CVD is the ankle brachial index (ABI), which is a sensitive and specific method for diagnosing peripheral arterial disease (PAD) and predicts cardiovascular events in the general population [14] [15] [16]. PAD is frequently asymptomatic and can be difficult to diagnose. ABI has facilitated the detection of subclinical PAD [14]. However, there are very limited data about abnormal ABI in patients with SLE [8] [17]. The aim of the present study was to compare ABI between SLE patients and controls.

\section{Patients and Methods}

\section{Study and controls population}

We conducted a case-control study in the Department of Internal Medicine of our institution during the period from August 1, 2017 to December 31, 2018.

We included patients followed for SLE in the departments of Internal Medicine, Nephrology and Dermatology that met the 1997 American College of Rheumatology (ACR) criteria [18].

SLE patients with other autoimmune diseases or a history of clinical cardiovascular events (such as myocardial infarction, stroke, or PAD) were not included.

Controls were recruited from volunteered subjects, age- and sex-matched, free 
of inflammatory rheumatism and a history of cardiovascular events.

\section{Clinical and laboratory assessment}

Patients with SLE and controls were assessed in a clinical research consultation.

The following data were refined in all subjects (patients and controls):

- general data such as age, gender, level of education, existence of smoking, alcohol consumption, family history of early cardiovascular disease among 1st degree relatives;

- the existence of a cardiovascular risk factor: smoking, overweight (defined by body mass index $>25 \mathrm{~kg} / \mathrm{m}^{2}$ ) or obesity (defined by a body mass index $>30$ $\mathrm{kg} / \mathrm{m}^{2}$ ), waist circumference measurement, high blood pressure (HBP), diabetes-mellitus (DM), dyslipidemia;

- the following biological investigations were carried out (CRP, creatinine and calculation of glomerular filtration rate, fasting blood glucose, total cholesterol, HDL cholesterol, LDL cholesterol, LDL cholesterol, LDL cholesterol, Uricemia).

In lupus patients, the following data were also specified:

- the disease duration, the diagnosis delay of SLE, the clinical pattern, the disease activity by the clinical systemic lupus erythematosus disease activity index (CSLEDAI) at the time of diagnosis and inclusion of this study, the duration of corticosteroid therapy, the daily dose and background therapy of SLE [19].

- biological explorations such as proteinuria have been carried out.

The immunological pattern was specified from the observation files.

\section{Ankle-brachial Index}

The ABI measurement was performed in all subjects after a 10-minute rest period. The brachial systolic pressure was recorded using a sphygmomanometer. The ankle pressure was recorded at the dorsal pedis artery and posterior tibial artery (the highest pressure in the limb was used) of the 02 lower limbs using a suitable sphygmomanometer cuff and a Doppler ultrasound velocity detector (Sonotrax ${ }^{*}$, pocket Doppler $8 \mathrm{MHz}$ ). The cuff was placed at the ankle level above the malleoli and the signal was detected by the Doppler pen. The average of the measurements was used. Abnormally ABI was defined by a value of 0.90 or less, or greater than 1.30. ABI value of 0.90 or less defined peripheral arterial occlusive disease (PAOD) and a value greater than 1.30 defined calcified vessels.

\section{Statistical analysis}

The data were recorded and analyzed using SPSS 23.0 software.

The results were expressed as a mean (+/- standard deviation) for the continuous variables and as percentages for the categorical variables.

Means were compared using $t$ Student test, and comparisons between categorical measures were made using chi 2 test. The $\mathrm{p}$-value $<0.05$ was considered statistically significant.

Ethics

The study was conducted in accordance with the Helsinki Declaration. An in- 
formed written consent has been obtained for all investigations on subject of the study.

\section{Results}

We included 100 subjects in our study, including 50 SLE patients and 50 control cases. This included 44 women and 06 men in patients with an identical distribution in controls. The mean age was $33.5 \pm 11.3$ years for cases and $33.3 \pm 11.3$ years with no statistically significant difference with a p-value of 0.93 .

All subjects were black Africans.

The mean duration of SLE was $60 \pm 59.3$ months and the mean diagnostic delay was $30.16 \pm 39.2$ months. The mean disease activity score assessed by the cSLEDAI score at the diagnosis was $12.15 \pm 6.8$. The mean disease activity score at the inclusion of the study was $6.59 \pm 5.6$.

Forty-six of patients (92\%) had corticosteroid therapy at the time of the evaluation. The mean duration of corticosteroid therapy at the time of the evaluation was $36.46 \pm 53.4$ months. The mean dose daily of corticosteroids (prednisone) was $22.61 \pm 18.9 \mathrm{mg} / \mathrm{d}$.

Forty-eight (96\%) patients were on hydroxychloroquine and 18 (36\%) SLE-lupus patients were also on immunosuppressive therapy including (cyclophosphamide, or mycophenolate, or azathioprine or Methotrexate).

The comparative analysis of the frequency of the different traditional cardiovascular risk factors in the two groups showed a higher frequency of cardiovascular risk factors such as dyslipidemia (p-value at 0.009), low level of serum HDL-cholesterol ( $\mathrm{p}$-value $<0.001$ ), hypertriglyceridemia ( $\mathrm{p}$-value at 0.000 ). Hyperuricemia ( $p$-value $<0.001$ ). Renal failure ( $p$-value at 0.043 ) was also correlated with SLE. Only the frequency of overweight/obesity was significantly higher in the control group (p-value at 0.028 ). There was no statistically significant difference in the frequency of factors such as diabetes-mellitus or fasting abnormal blood sugar (p-value: 0.988 ), smoking (p-value: 0.79 ) and high blood pressure (p-value: 1 ). Table 1 summarized the risk cardiovascular factors in patients with SLE and controls.

In patients, the mean $\mathrm{ABI}$ was $1.16 \pm 0.45$ and in the control group at $1.08 \pm$ 0.45 without significant difference. The abnormally ABI was recorded in $19 \mathrm{pa}-$ tients with SLE (38\%) and 08 controls (16\%) with a significant difference (p-value: 0.01 and odds ratio: 3.22). Eight patients (16\%) and four controls (8\%) had low ABI without significant difference (p-value: 0.11 but odds-ratio at 2.98). Eleven patients with SLE (22\%) and five controls (10\%) had high ABI (p-value: 0.05 and odds-ratio: 3.24). The values of ABI in patients with SLE and controls were summarized in Table 2.

In the group of patients with SLE, only the disease activity score (cSLEDAI) at the inclusion of the present study was correlate to abnormal ABI.

Any other clinical data in the time of the diagnostic and inclusion, or cardiovascular risk factors, lupus specific related factors and corticosteroid therapy were correlated with ABI abnormalities (Tables 3-6). 
Table 1. Cardiovascular risk factors in patients and controls.

\begin{tabular}{|c|c|c|c|}
\hline Parameters & Cases & Controls & p-value \\
\hline \multicolumn{4}{|l|}{ Age } \\
\hline Mean age & $33.5(11.3)$ & $33.3(11.3)$ & 1 \\
\hline \multicolumn{4}{|l|}{ Sex } \\
\hline Sex-ratio & 0.13 & 0.13 & 1 \\
\hline Women & $44(88 \%)$ & $44(88 \%)$ & 1 \\
\hline Men & $6(12 \%)$ & $6(12 \%)$ & 1 \\
\hline Tobacco & $3(6 \%)$ & 0 & 0.79 \\
\hline No exercice & $3(6 \%)$ & $1(2 \%)$ & 0.30 \\
\hline Alcohol & $1(2 \%)$ & 0 & 0.31 \\
\hline Familial history of early CVD & 0 & 0 & NA \\
\hline Familial Diabetes-mellitus & 13 & 20 & 0.13 \\
\hline CVD & 0 & 0 & NA \\
\hline Obesity & $4(8 \%)$ & $6(12 \%)$ & 0.56 \\
\hline Mean BMI $\left(\mathrm{kg} / \mathrm{m}^{2}\right)$ & 21.27 & 23.8 & 0.01 \\
\hline \multicolumn{4}{|l|}{ Waist circumference } \\
\hline Mean value & 77.53 & 82.38 & 0.11 \\
\hline Abnormal & $11(22 \%)$ & $22(44 \%)$ & 0.15 \\
\hline Hypertension & $6(12 \%)$ & $6(12 \%)$ & 1 \\
\hline Diabetes-mellitus & $2(4 \%)$ & $1(2 \%)$ & 0.15 \\
\hline \multicolumn{4}{|l|}{ Fasting serum glucose } \\
\hline Abnormal value & $2(4 \%)$ & $1(2 \%)$ & 0.98 \\
\hline Dyslipidemia & $31(62 \%)$ & $18(54 \%)$ & 0.01 \\
\hline \multicolumn{4}{|l|}{ Cholesterol } \\
\hline Mean (g/l) & 1.97 & 1.98 & 0.95 \\
\hline Abnormal value & $14(28.5 \%)$ & $13(26 \%)$ & 0.82 \\
\hline \multicolumn{4}{|l|}{ HDL cholesterol } \\
\hline Mean (g/l) & 0.52 & 0.61 & 0.02 \\
\hline Abnormal & $13(26.53 \%)$ & 1 & 0.00 \\
\hline \multicolumn{4}{|l|}{ LDL cholesterol } \\
\hline Mean $(g / l)$ & 1.1 & 1.3 & 0.10 \\
\hline Abnormal & $15(30.6 \%)$ & $22(44 \%)$ & 0.14 \\
\hline \multicolumn{4}{|l|}{ Triglycerides } \\
\hline Mean (g/l) & 1.53 & 0.62 & 0.00 \\
\hline Abnormal & $17(34.6 \%)$ & 0 & 0.00 \\
\hline \multicolumn{4}{|l|}{ Uricemia } \\
\hline Mean (mg/l) & 62.18 & 46.49 & 0.00 \\
\hline Abnormal & $24(48.9 \%)$ & $3(6 \%)$ & 0.00 \\
\hline Renal failure & $4(8.1 \%)$ & 0 & 0.04 \\
\hline Mean GFR (1/min) & 141.5 & 123.73 & 0.01 \\
\hline Overweight/obesity & $11(22 \%)$ & $20(40 \%)$ & 0.03 \\
\hline
\end{tabular}

CVD: cardiovascular disease BMI: Body Mass Index, HDL: High Density Lipoprotein, LDL: Low Density Liprotein, GFR: glomerular filtration rate. 
Table 2. Ankle brachial index in patients with SLE and controls.

\begin{tabular}{ccccc}
\hline ABI & Cases & Controls & p-value & Odds-ratio (IC: 95\%) \\
\hline ABI, normal-value & & & & NS \\
$($ ABI: $>0.9$ and $\leq 1.3)$ & $31(62 \%)$ & $42(84 \%)$ & NS & 3.22 \\
Abnormal ABI & & & & 2.98 \\
ABI $\leq 0.9$ or $>1.3$ & $19(38 \%)$ & $8(16 \%)$ & 0.01 & 3.24 \\
PAOD (ABI $\leq 0.9)$ & $8(16 \%)$ & $3(6 \%)$ & 0.11 & 0.05 \\
Calcified vessels $(\mathrm{ABI}>1.3)$ & $11(22 \%)$ & $5(10 \%)$ & 05 \\
\hline
\end{tabular}

ABI: Ankle brachial index, NS: non significant. PAOD: peripheral arterial occlusive disease.

Table 3. Correlation between organ-involvement (at the diagnostic) and ankle brachial index.

\begin{tabular}{cccc}
\hline Pattern clinic & Abnormal ABI & Normal ABI & p-value \\
\hline Muco-cutaneous & 12 & 23 & 0.40 \\
Arthralgia/Arthritis & 17 & 26 & 0.57 \\
NPSLE & 3 & 6 & 0.71 \\
LN & 7 & 20 & 0.08 \\
Hematological SLE & 15 & 21 & 0.39 \\
Serositis & 4 & 9 & 0.63 \\
General signs & 10 & 25 & 0.06 \\
\hline
\end{tabular}

ABI: ankle brachial index NPSLE: Neuropsychiatric systemic lupus erythematosus SLE: systemic Lupus erythematosus LN: Lupus Nephritis.

Table 4. Correlation between organ-involvement (at the inclusion) and ankle brachial index.

\begin{tabular}{cccc}
\hline Pattern clinic & Abnormal ABI & Normal ABI & p-value \\
\hline Mucocutaneous & 11 & 21 & 0.48 \\
Arthralgia/Arthritis & 8 & 13 & 0.99 \\
NPSLE & 2 & 3 & 0.92 \\
LN & 5 & 15 & 0.12 \\
Hématological SLE & 12 & 19 & 0.89 \\
\hline
\end{tabular}

ABI: ankle brachial index NPSLE: Neuropsychiatric systemic lupus erythematosus SLE: systemic Lupus erythematosus LN: Lupus Nephritis.

Table 5. Correlation between ABI and cardiovascular risk factors in patients with SLE.

\begin{tabular}{cccc}
\hline Factors & Abnormal ABI & Normal ABI & p-value \\
\hline Age & 34.79 & 32.77 & 0.349 \\
Men & 1 & 5 & 0.251 \\
Dyslipidemia & 13 & 18 & 0.435 \\
Hyperuricemia & 9 & 15 & 0.858 \\
Renal failure & 1 & 3 & 0.555 \\
Tabacco & 1 & 2 & 0.864 \\
No exercice & 1 & 2 & 0.864 \\
Overweight/Obesity & 5 & 6 & 0.606 \\
Hypertension & 2 & 4 & 0.802 \\
Diabetes-mellitus & 0 & 2 & 0.258 \\
\hline
\end{tabular}

ABI: Ankle Brachial Index. 
Table 6. Correlation between ABI and SLE-related factors.

\begin{tabular}{cccc}
\hline SLE-related factors & Abnormal ABI & Normal ABI & p-value \\
\hline Disease duration & 71.21 & 53.94 & 0.32 \\
Diagnostic delay & 44.06 & 22.10 & 0.05 \\
cSLEDAI (diagnostic) & 10.93 & 13.08 & 0.34 \\
Steroid dose daily (mg) & 20.53 & 24.07 & 0.53 \\
Cumulative dose (mg) & 1374.3 & 544.5 & 0.29 \\
Steroid duration & 43.32 & 32.14 & 0.47 \\
CRP & 12 & 11 & 0.05 \\
Proteinuria & 8 & 18 & 0.22 \\
LN & 6 & 13 & 0.27 \\
cSLEDAI (mean value at inclusion) & 7.49 & 3 & 0.004 \\
\hline
\end{tabular}

SLE: Systemic Lupus Erythematosus, ABI: Ankle Brachial Index, cSLEDAI: Clinical Systemic Lupus Erythematosus Disease Activity Index CRP: C-Reactive Protein. LN: Lupus Nephritis.

\section{Discussion}

SLE is one of the common systemic auto-immune disease also called connective tissue-disease [20] [21].

SLE has been associated with accelerated atherosclerosis and increased risk of cardiovascular disease [2]-[7]. Thus, in different series, the prevalence of symptomatic coronary artery disease varied from $6 \%$ to $10 \%$. Likewise, the risk of hospitalization for stroke has been shown to be 2-fold higher in patients with SLE in comparison with the general population [22].

Urowitz et al., described also a bimodal pattern of mortality in SLE with a late peak of mortality, after 5-years of follow-up, mainly due to atherosclerosis [23].

Different modalities have been used to evaluate subclinical atherosclerosis, such as evaluating carotid intima-media thickness by ultrasound, measuring coronary calcium score by cardiac imaging and ankle-brachial index for peripheral vascular disease [11] [12] [13].

Whether atherosclerosis in coronary artery, has been widely studied in the literature, peripheral arterial disease has been infrequently described [8] [17]. We reported, in our best knowledge, we reported the first study in sub-Saharan Africa, who compared ABI in patients with SLE between controls.

There are also paucity data in the international literature. Tziomalos et al., reported in 2016, only four similar studies prior to their publication about the prevalence and the determinants of abnormal ABI in patients with SLE. Three of these previous studies did not include a control population [8].

In the present study, we found that SLE-patients have a higher prevalence of abnormal ABI compared with age- and sex-matched controls.

Hassan et al., showed in their case control study including 120 patients with SLE and 100 volunteered persons a prevalence of PAD at $25 \%$ (versus $6 \%$ in controls) with a significant difference (p-value $<0.001)$ [2]. June and Scalzi reported also a higher percentage of SLE patients with PAD than controls $(33 \%$ 
versus $20 \%$ ) with a statistically significant difference [11]. However, Tziomalos et al., didn't find a statistic difference in the prevalence of PAD between SLE patients and controls (10\% versus $5.4 \%$ p-value $=$ NS) [8].

Nevertheless, there is difference in definition of ABI in these studies. Hassan et al. and June et al., applied a cut-off ABI value of 1.00 while Tziomalos used a cut-off value of 0.90 according to the current recommendations [14].

In our study, we recognized abnormal ABI with two subgroups, peripheral arterial occlusive disease (low ABI cut-off value 0.90) and no-compressible calcified vessels $(\mathrm{ABI}>1.3)$ [15].

Our findings, about low ABI, similar to those of Tziomalos et al., suggest that our studies had limited power to identify significant differences between patients with SLE and controls due to the small number of patients and controls and the narrow range of ABI values [8]. The prevalence of PAOD could be also underestimated by the higher frequency of abnormally high ABI. Vascular calcification doesn't imply that occlusive lesions are present, although these two conditions frequently coexist [14]. When vascular calcification is present, however, stenotic disease can't be detected by the ABI [14].

We didn't find any study about SLE and abnormally high ABI value. This condition was described in diabetes mellitus or end stage renal which are associated with an increasing of cardiovascular risk [14].

Accelerated atherosclerosis in SLE has been related to traditional risk factors and some SLE related factors such as disease duration, steroid therapy, activity, organ damage [5] [8] [9] [10]. In previous studies, the major determinants of low ABI were age and smoking. June and Scalzi reported also a link with low $\mathrm{ABI}$ and African-American black ethnicity.

In the present study, only the disease activity at the inclusion of study was correlated to the abnormal ABI. This finding may suggest that chronic inflammation plays an important role in the genesis of atherosclerosis in patients with SLE.

We didn't find any correlate with other clinical and laboratory parameters, such as cardiovascular risk factors.

All of patients were African-black and ninety-six percent of them were taking antimalarials drugs which make it very difficult to analyze the effect of these factors.

The main limitation of our study was the small number of cases and controls. The measurement of thrombotic variables (such as plasma fibrinogen, von-Willebrand factor antigen (vWF), tissue plasminogen activator antigen (tPA) and fibrin D-dimer or antiphospholipid antibodies) was not available.

Identifying subclinical PAD gives an opportunity to provide primary and secondary prevention potentially before clinical event. PAS is associated with a high frequency of vascular disease in other territories, such as coronary (4-fold higher risk) and cerebral arteries (2 to 3 -fold increased risk of stroke). PAD is also associated with a 3-fold increasing mortality [22]. ABI is also a cost-effective method for the diagnostic of PAD especially in our developing countries. 


\section{Conclusions}

In conclusion, this study showed an increasing prevalence of abnormal ABI in black African patients with SLE compared to controls.

We found also a correlation between disease activity and abnormal ABI that highlights the importance of the chronic inflammation in the genesis of atherosclerosis in SLE and the need to induce remission in the management of SLE.

However, further studies are needed in sub-Saharan Africa, to evaluate other potential factors that may be induced premature atherosclerosis in patients with SLE.

\section{Consent for Publication}

Written informed consent for publication was obtained from the patients.

\section{Funding}

The authors declare that they have not received another source of funding.

\section{Conflicts of Interest}

The authors declare no conflicts of interest regarding the publication of this paper.

\section{References}

[1] Felten, R., Dervoric, E., Chasset, F., Gottenberg, J.E., Sibilia, J., Scher, F. and Arnaud, L. (2018) The 2018 Pipeline of Targeted Therapies under Clinical Development for Systemic Lupus Erythematosus: A Systematic Review of Trials. Autoimmunity Reviews, 17, 781-790. https://doi.org/10.1016/j.autrev.2018.02.011

[2] Hassan, A.A., Habib, H.M. and Eissa, A.A. (2013) Peripheral Arterial Disease in Patients with Systemic Lupus Erythematosus. International Journal of Rheumatic Diseases, 16, 319-324. https://doi.org/10.1111/1756-185x.12025

[3] Croca, S. and Rahman, A. (2017) Atherosclerosis in Systemic Lupus Erythematosus. Best Practice \& Research: Clinical Rheumatology, 31, 364-372. https://doi.org/10.1016/j.berh.2017.09.012

[4] Freri, M. and Stampfl, H. (2016) Systemic Lupus Erythematosus and Atherosclerosis: Review of the Literature. Autoimmunity Reviews, 15, 16-21. https://doi.org/10.1016/j.autrev.2015.08.007

[5] Mayra, G. and Mavragani, C.P. (2017) Cardiovascular Disease in Systemic Lupus Erythematosus: A Comprehensive Update. Journal of Autoimmunity, 82, 1-12. https://doi.org/10.1016/j.jaut.2017.05.008

[6] Petri, M.A., Barr, E. and Magder, L.S. (2019) Development of a Systemic Lupus Erythematosus Cardiovascular Risk Equation. Lupus Science \& Medicine, 6, e000346. https://doi.org/10.1136/lupus-2019-000346

[7] McMahon, M., Skaggs, B., Grossman, J., Wong, W.K. and Sahakian, L. (2019) Comparison of Predicts Atherosclerosis Biomarkers Changes after Initiation of New Treatments in Patients with SLE. Lupus Science \& Medicine, 6, e000321. https://doi.org/10.1136/lupus-2019-000321

[8] Tziomalos, K., Gkougkourelas, I., Sarantopoulos, A., Bekiari, E., Makri, E., Raptis, N., et al. (2017) Arterial Stiffness and Peripheral Arterial Disease in Patients with 
Systemic Lupus Erythematosus. Rheumatology International, 37, 293-298. https://doi.org/10.1007/s00296-016-3610-4

[9] Yang, D.H., Leong, P.Y., Sia, S.K., Wang, Y.H. and Wei, J.C. (2019) Long-Term Hydroxyxhloroquine Therapy and Risk of Coronary Artery Disease in Patients with Systemic Lupus Erythematosus. Journal of Clinical Medicine, 5, Pii: E796. https://doi.org/10.3390/jcm8060796

[10] Mercurio, V., Lobasso, A., Barbieri, L., Parella, P., Ciervo, D., et al. (2019) Inflammatory, Serological and Vascular Determinants of Cardiovascular Disease in Systemic Lupus Erythematosus Patients. International Journal of Molecular Sciences, 20, 2154. https://doi.org/10.3390/ijms20092154

[11] June, R.R. and Scalzi, L.V. (2013) Peripheral Vascular Disease in Systemic Lupus Patients. Journal of Clinical Rheumatology, 19, 367-372. https://doi.org/10.1097/RHU.0000000000000017

[12] Jung, J., Kim, H., Lee, H.Y. and Suh, C. (2019) Body Mass Index and Glucocorticoid Dose Contribute to Atherosclerosis in Korean Patients with Systemic Lupus Erythematosus. A Prospective 4 Years Follow-Up Study. International Journal of Rheumatic Diseases, 22, 1410-1418. https://doi.org/10.1111/1756-185X.13588

[13] Roldan, P.C., Greene, E.R., Qualls, C.R., Sibbitt, W.L. and Roldan, C.A. (2019) Progression of Atherosclerosis versus Arterial Stiffness with Age within and between Arteries in Systemic Lupus Erythematosus. Rheumatology International, 39, 1027-1036. https://doi.org/10.1007/s00296-019-04267-y

[14] Aboyans, V., Criqui, M.H., Abraham, P., Allison, M.A., Creager, M.A., Diehm, C., et al. (2012) Measurement and Interpretation of Ankle-Brachial Index. A Scientific Statement from the American Heart Association. Circulation, 126, 2890-2909. https://doi.org/10.1161/CIR.0b013e318276fbcb

[15] Hossain, P., Kokkinidis, D.G. and Armstrong, E.J. (2019) How to Assess a Claudication and When to Intervene. Current Cardiology Reports, 21,138. https://doi.org/10.1007/s11886-019-1227-4

[16] Hong, J.B., Leonards, C.O., Endres, M., Siegerink, B. and Liman, T.G. (2016) Ankle-Brachial Index and Recurrent Stroke Risk: Meta-Analysis. Stroke, 47, 317-322. https://doi.org/10.1161/STROKEAHA.115.011321

[17] Chuang, Y.W., Yu, M.C., Lin, C.L., Yu, T.M., Shu, K.H. and Kao, C.H. (2015) Risk of Peripheral Arterial Occlusive Disease in Patients with Systemic Lupus Erythematosus. Medicine, 94, 1-7. https://doi.org/10.1097/MD.0000000000002121

[18] Hochberg, M.C. (1997) Updating the American College of Rheumatology Revised Criteria for the Classification of Systemic Lupus Erythematosus. Arthritis \& Rheumatology, 40, 1725. https://doi.org/10.1002/art.1780400928

[19] Parodis, I., Emamika, S., Gomez, A., Gunnarson, I., Van Vollenhoven, R.F. and Chatzidioniysiou (2019) Clinical SLEDAI-2K Zero May Be a Pragmatic Outcome Measure in SLE Studies. Expert Opinion on Biological Therapy, 19, 157-168. https://doi.org/10.1080/14712598.2019.1561856

[20] Dorner, T. and Furie, R. (2019) Novel Paradigms in Systemic Lupus Erythematosus. The Lancet, 393, 2344-2358. https://doi.org/10.1016/S0140-6736(19)30546-X

[21] Cervera, R., Khamashta, M.A., Font, J., Sebastiani, G.D., Gil, A., Lavilla, P., et al. (1993) Systemic Lupus Erythematosus: Clinical and Immunologic Patterns of Disease Expression in a Cohort of 1000 Patients. Medicine, 72, 113-124. https://doi.org/10.1097/00005792-199303000-00005

[22] Erdozain, J.G., Villar, I., Nieto, J. and Ruiz-Irastorza (2014) Peripheral Arterial Disease in Systemic Lupus Erythematosus: Prevalence and Risk Factors. The Journal of 
Rheumatology, 41, 310-317. https://doi.org/10.3899/jrheum.130817

[23] Urowitz, M.B., Bookman, A.A., Koehler, B.E., Smythe, H.A. and Ogryzlo, M.A. (1976) The Bimodal Mortality Pattern of Systemic Lupus Erythematosus. The American Journal of Medicine, 60, 221-225.

https://doi.org/10.1016/0002-9343(76)90431-9 